\title{
Plasma Thrusters for In-Space Propulsion; New Trends and Physical Limitations
}

\author{
Dmitry Dyubo (D), Jorge González, Oleg Tsybin (D), and Luis Conde
}

\begin{abstract}
In-space plasma propulsion is today a necessity for the economic competitiveness of commercial satellites. In plasma thrusters the impulse is imparted by a mesothermal plasma jet where ions drift a supersonic velocities. Compact multielectrode systems for ion acceleration are studied by a combination of analytical calculations and computer simulations. The results show exahust speeds of $140 \mathrm{~km} / \mathrm{s}$ can be reached with specific impulses higher than unsual plasma thrusters. Additionally, the ion velocity distribution functions of the plasma jet exhasut obtained with retarded field energy analyzers that can be used to cross validate the results of numerical simulations.
\end{abstract}

Keywords Plasma thrusters $\cdot$ Ion acceleration $\cdot$ Space propulsion

D. Dyubo $(\varangle) \cdot$ O. Tsybin

Institute of Physics, Nanotechnology and Telecommunications, Peter the Great St. Petersburg Polytechnic University, Polytechnicheskaya 29, 195251 St. Petersburg, Russia

e-mail: dyubo.db@edu.spbstu.ru

O. Tsybin

e-mail: otsybin@ rphf.spbstu.ru

J. González

DIFFER-Dutch Institute for Fundamental Energy Research, De Zaale 20, 5612 AJ Eindhoven, The Netherlands

e-mail: J.GonzalezMunos@differ.nl

L. Conde

Department of Applied Physics, ETSI Ingeniería Aeronáutica y del Espacio, Universidad

Politécnica de Madrid, 28040 Madrid, Spain

e-mail: 1conde@aero.upm.es 


\subsection{Introduction}

In-space electric propulsion technologies have today become a necessity for the economic competitiveness of commercial satellites. As the usual chemical engines, the electric thrusters operate according to the basic principle of action and reaction where the exhaust gas velocity $v_{e x}$ drives the thrust $F=\dot{m} v_{e x}$ delivered. The electric propulsion makes use of electricity to heat and/or ionize a gas to achieve much higher $v_{\text {ex }}$ propellant velocities than conventional chemical thrusters [1-4].

Specifically, plasma thrusters expel an electron-ion plasma stream, usually called plasma plume, where heavy positive particles are accelerated by externally imposed or self-consistent plasma electric fields. Such devices are essentially ion accelerators where the motion of heavy charged particles is focused along a fixed direction and their basic characteristics are shown in Table 7.1.

Additionally, an efficient plasma thruster design requires supersonic ion exhaust velocities, similarly to the fast motion of a solid body in a neutral gas. In the frame of the moving object the gas flows faster than pressure fluctuations produced by short range collisions of molecules that propagate at sound speed. Likewise, ion density perturbations $\delta n_{i}$ in plasmas are transmitted at the ion sound speed, $c_{i s}=$ $\left.\left[k_{B}\left(T_{e}+T_{i}\right) / m_{i}\right)\right]^{1 / 2}$ where $T_{e}$ is the electron temperature and $m_{i}$ the ion mass [5]. Basically, density perturbations are produced by the thermal ion motion that create local electric fields, these are shielded by the more mobile electrons on a faster time scale. Hence, collisionless compressions and rarefractions propagate in a plasma similarly to sound waves in ordinary gases.

For the plasma thrusters of Table 7.1 the electron temperatures are in the range $T_{e} \sim 1-50 \mathrm{eV}$ much higher than ion temperature $T_{e} \gg T_{i} \sim 0.025 \mathrm{eV}$ and these conditions the ion thermal speed $v_{T i}=\sqrt{2 k_{B} T_{i}} / m_{i}<c_{i s}$ is much slower that the ion acoustic speed. The characteristic velocities of heavy particles in the plasma exhaust stream are,

$$
v_{T i} \ll c_{i s}<v_{e x} \ll v_{T e}
$$

where $v_{T e}=\sqrt{2 k_{B} T_{e} / m_{e}}$ is the electron thermal speed. This plasma flow where ions move along a fixed direction with supersonic velocities $v_{e x}>c_{i s}$ is called mesothermal and a Mach number $M_{o}=v_{e x} / c_{i s}>1$ can be introduced for the motion of ions.

Similarly, the Earth's ionospheric plasma is colder $\left(T_{i} \sim T_{e} \simeq 0.05 \mathrm{eV}\right)$ than laboratory plasmas, but orbital speed of vehicles is high, about $v_{o} \sim 6-8 \mathrm{~km} / \mathrm{s}$ in low Earth orbit. Thus, for orbiting satellites we also have $v_{T i} \ll v_{o} \ll v_{T e}$ and the Mach number in this case $M_{o}=v_{o} / c_{i s}$ involves their orbital speed.

The mesothermal plasma flow is achieved in plasma thrusters by multi-electrode configurations biased at high potentials in gridded ion engines and/or by selfconsistent plasma electric fields in Hall-effect and multi-cusp thrusters [1-4]. The Table 7.1 shows typical values of thrust and ion exhaust velocities as well as the specific impulses $I_{s p}=v_{e x} / g_{o}$ where $g_{o}$ is the standard Earth's acceleration. 
Table 7.1 Performance data for plasma thrusters from references [2, 6, 7]. The acronyms are: ECR, electron cyclotron resonance thruster; GIT, Gridded ion engine; HET, Hall effect thruster and HEMPT, highly efficient multistage plasma thruster.

\begin{tabular}{l|l|l|l|l|l}
\hline Thruster & $v_{e x}(\mathrm{~km} / \mathrm{s})$ & Thrust $(\mathrm{mN})$ & $I_{s p}(\mathrm{~s})$ & Power $(\mathrm{kW})$ & Propellant \\
\hline Helicon & 5.0 & 1.0 & $\sim 500$ & 0.1 & $\mathrm{Ar}, \mathrm{Kr}, \mathrm{Xe}$ \\
ECR & 10.0 & 1.0 & 1000 & 0.03 & $\mathrm{Xe}$ \\
GIT & 29.0 & $1-100$ & 3000 & $0.1-5$ & $\mathrm{Xe}$ \\
HET & 15 & $10-1000$ & 1500 & $0.2-20$ & $\mathrm{Xe}$ \\
HEMPT & $15-30$ & $8-250$ & $1500-3000$ & $5-6$ & $\mathrm{Xe}$ \\
\hline
\end{tabular}

In principle, there is no theoretical limit to the speed that ions accelerated by a stationary electric field can reach. Unfortunately, it is limited by practical considerations such as weight, the characteristic size of the equipment, electrical energy consumption, etc.

For ions to reach supersonic speeds an efficient momentum transference mechanism is required such as multi-electrode structures where ions gain energy moving along an acceleration channel. In this paper different configuration of these structures for ion acceleration are investigated using a combination of analytical calculations and computer simulations. As we shall see compact multi-electrode systems where mesothermal plasma flow exhaust can exceed the limits of Table 7.1 can be designed.

\subsection{Numerical Simulations}

Computer simulations save time and resources since the numerical assessment of plasma thruster performance save considerable workshop effort and expensive laboratory testing. Specifically, the configuration of accelerating electrodes as these impart to ions their exhaust velocity, which is critical for delivered thrust level and the thruster electrical efficiency [1,2].

The study of electron and ion motion in the inter-electrode space is difficult as the self-consistent electric field results from the interaction of moving charged particles and metallic electrodes that are electrically connected to fixed electric potentials. Then, computer models for the electric field and particle trajectories using Computer Simulation Technology (CST) software [8], combined with analytical algorithms have been employed to estimate the capabilities and mechanical force delivered by different ion accelerator configurations [9]. This approach also allows to evaluate the different physical parameters involved as well as the performance of different propellants.

In our simulations the ions that are produced by different means and enter in the ion injection plane (see Fig. 1 in Ref. [9]), moving along the cylindrical acceleration channel created by circular holes in a set of superposed electrodes. The self-consistent electromagnetic field focus the ion trajectories increasing their velocity as they move 


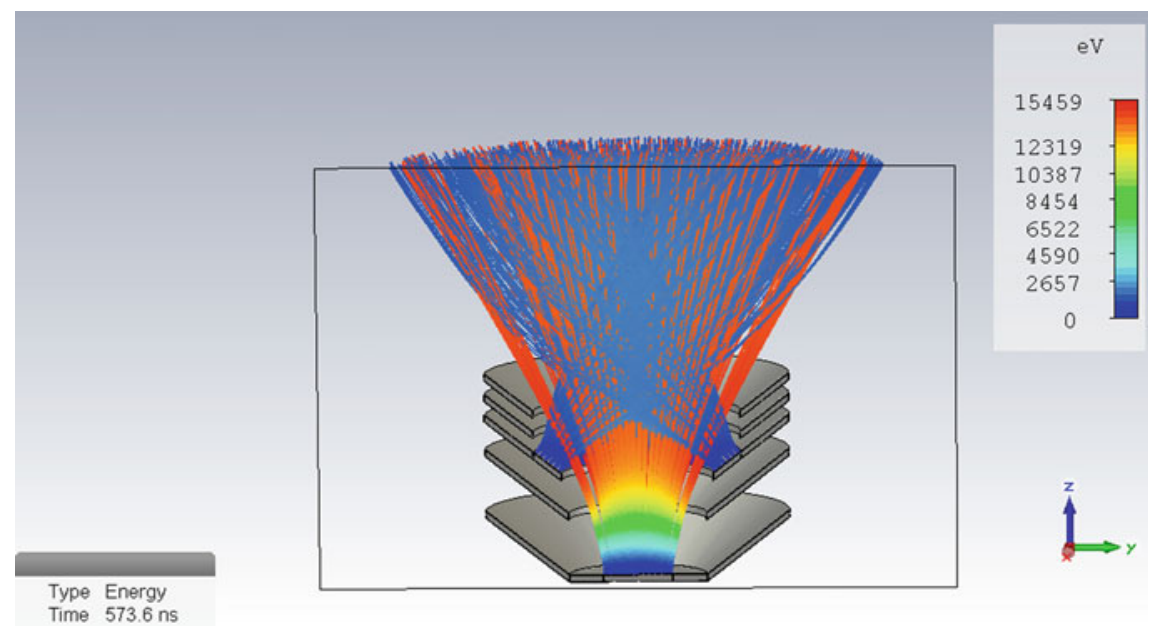

Fig. 7.1 Schematic illustration of a multi-electrode optimized structure with the ion source placed at is basement. The electron and ion trajectories are represented by straight lines and the color code bar indicates the energies of charged particles, $I_{e}=100 \mathrm{~mA}$ and $I_{i}=14 \mathrm{~mA}$.

downstream along the thrust axis, parallel to the $Z$ axis of symmetry. Different configurations has been investigated to optimize the thrust imparted by a multielectrode system and Figs. 7.1 and 7.2 show two of these structures.

The neutral gas pressure for thruster operation in the outer space is very low, so the typical dimension of inter-electrode spaces is much smaller than the mean free path for collisions between neutral atoms and charged particles. Then, ion and electron motion along the acceleration channel is collisionsless and basically governed by electromagnetic forces. Additionally, the ion flow should be neutralized, otherwise the extracted current downstream is limited by space-charge effects. The electron neutralizer of accelerated cations was constructed on the principle of combined beams and a virtual cathode, which is basically similar to those on ion-plasma thrusters. The resulting electron-ion plasma moves along the acceleration channel where $I_{e}$ and $I_{i}$ are the electron and ion current transported.

The thrust force $F_{T i}$ can be obtained by integration along the trajectories of the ion mass $d m(z)$ within the sheet of thickness $d z$ that moves at speed $v(z)$ as,

$$
F_{T i}\left(Z_{n}\right)=\int_{0}^{Z_{n}} \frac{d v(z)}{d t} d m(z)
$$

Equivalently, this force can also be calculated as the force exerted by the electric field $E(z)$ on the small sheet of electric charge $d Q(z)$;

$$
F_{T i}\left(Z_{n}\right)=\int_{0}^{Z_{n}} E(z) d Q(z)
$$




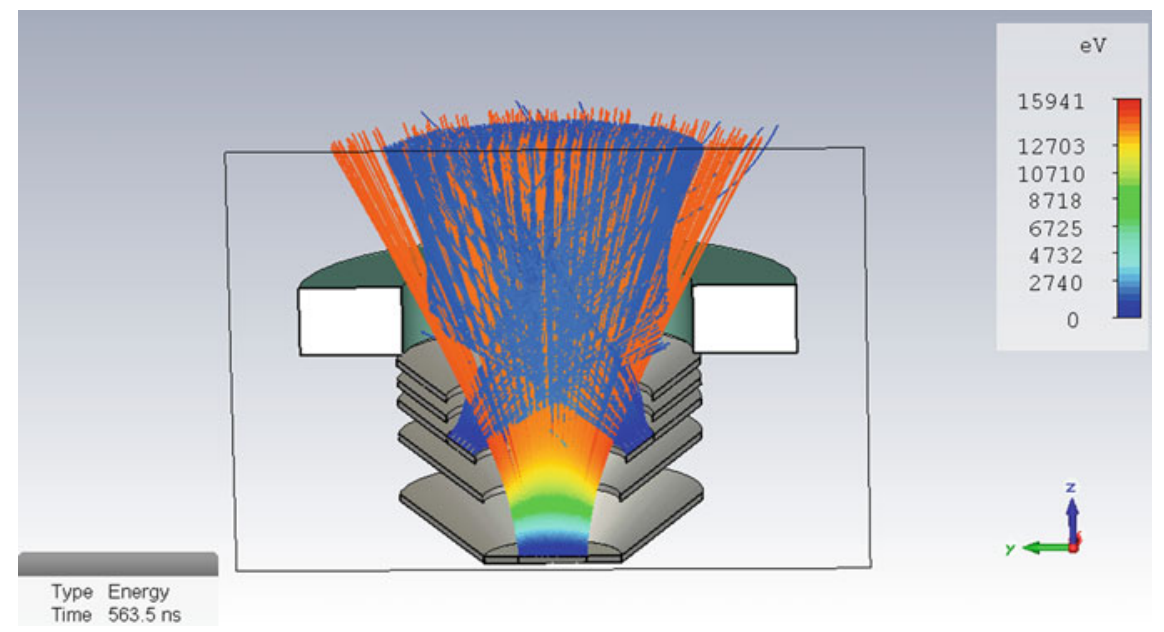

Fig. 7.2 Schematic illustration of a multi-electrode optimized structure with the ion source placed at is basement and a ring magnet at the top. The electron and ion trajectories are represented by straight lines and the color code bar indicates the energies of charged particles, $I_{e}=100 \mathrm{~mA}$ and $I_{i}=16 \mathrm{~mA}$.

The upper limit $Z_{n}$ in both integrals corresponds to the transition of ions from an acceleration area to a neutralization area. Assuming the time scale for the neutralization process at $z=Z_{n}$ is much faster than the characteristic time for ion motion we have $d v(z) / d t=0$ and $E(z)=0$ and,

$$
\int_{Z_{n}}^{\infty} E(z) d Q(z)=0
$$

The thrust is only produced in the ion acceleration area so $F_{T i}\left(z>Z_{n}\right)$ is constant as shows Fig. $7.4 \mathrm{~b}$ below.

The multi-electrode ion acceleration structure of Figs. 7.1 and 7.2 the electron and ion trajectories are shown as lines and the side color code bar corresponds to the energies of charged particles, that are within $0-15 \mathrm{keV}$ range. The shape and size of electrodes, their polarization potentials, the ion emission area and the magnitude of charged particle currents were optimized in successive numerical simulations. In particular, the physical conditions under which the electron beam creates an effective potential well for acceleration of ions were determined.

The ion source is placed at its bottom with an active circular area of $10 \mathrm{~mm}$ in diameter. Next, are disposed four plane electrodes separated 8, 4, 2 and $2 \mathrm{~mm}$ (from bottom to up) perpendicular to the positive $Z$ axis of symmetry. The open channel for ion acceleration is made by aligned circular holes in the plane electrodes of 10 , 11,15 and $15 \mathrm{~mm}$ radii respectively.

In Fig. 7.1 the ion current is $I_{i}=14 \mathrm{~mA}, I_{e}=100 \mathrm{~mA}$ and the four lower electrodes are electrically biased to $15.0,-0.5,-1,0.5 \mathrm{kV}$ and the upper external electrode 


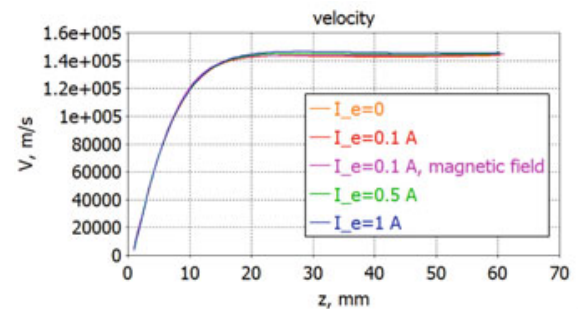

(a) The axial ion velocity.

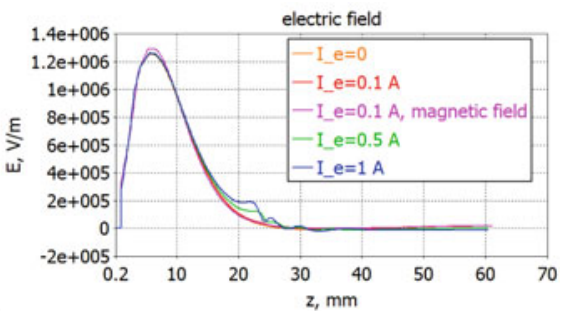

(b) The axial electric field.

Fig. 7.3 Numerical calculation along the $Z$ axis of symmetry in the multi-electrode models of Figs. 7.1 (red line) and 7.2 (green line).

is grounded. The numerical simulations show the ions achieve high energies with a moderate divergence of the plasma stream with respect to the symmetry axis.

The second structure of Fig. 7.2 shows a permanent ring magnet of $\mathrm{B}=70 \mathrm{mT}$ magnetic flux density added over the exit section of the multi-electrode system. As it can be appreciated, its effects is to improve the divergence of the ion trajectories with respect to Fig. 7.1. The separation of electrodes were 8, 4, 2 and $2 \mathrm{~mm}$ in this case whereas hole radii and electrode polarization potentials were as in previous Fig. 7.1.

The ion velocity along the $Z$ thrust axis is shown in Fig.7.3a that is abruptly incremented along the first $20 \mathrm{~mm}$ of the multi-electrode structure to reach a $v_{e x} \simeq$ $140 \mathrm{~km} / \mathrm{s}$ constant exhaust speed. The magnetic field has little effect in this case as ions essentially move parallel to the $Z$ axis and are weakly affected by the magnetic force. Thus, they leave the multi-electrode acceleration area and reach a constant exhaust speed. However, ions with a radial component of velocity result confined in the direction of the thrust axis by the axial magnetic field as seen by comparing Figs. 7.1 and 7.2. Hence, the divergence of the plasma plume is reduced.

The electric field on Fig. 7.3b is responsible for ion acceleration and it has an sharp peak approximately located close to the first electrode of Figs.7.1 and 7.2. The electric field is obviously not affected by the magnetic field and decreases fast outside the structure and shows a smooth profile and low values in the expanding plasma plume.

The Fig. 7.4a shows the axial electric potential profile along the $Z$ axis of the simulation. The abrupt increment in the electric potential extends to negative values of the coordinate as an electric field is necessary to extract the ions from the source. This field is the simulation boundary condition for $z \lesssim 0$ before the injection plane at $z=0$ (see Fig. 1 in Ref. [9]).

The electric potential presents a sharp peak in Fig. 7.3b in the region where the electric field increments and next a fast decrease, as the electron neutralization of the ion beam takes place. For axial distances outside of the multi-electrode structure the potential profile becomes smoother as the plasma plume expands in a vacuum. The effect of the magnetic field at the exit section is to reduce the divergence of the plasma plume, so plasma flow becomes denser. This effect is observed Fig. 7.4b by 


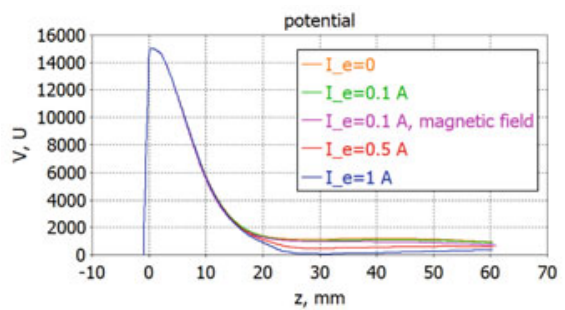

(a) The electric potential.

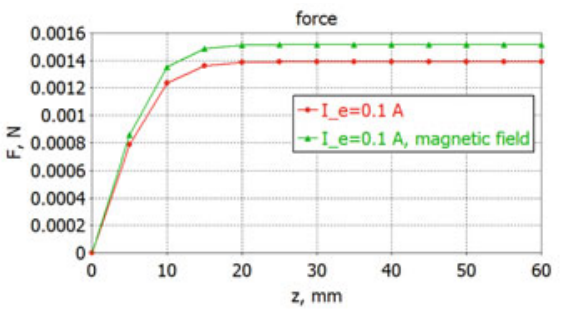

(b) The axial force $F_{T i}(z)$.

Fig. 7.4 The force and electric potential along the $Z$ axis of symmetry in the multi-electrode models of Figs. 7.1 (red line) and 7.2 (green line).

the increased electric potential for $z>20$ outside the multi-electrode structure when the axial magnetic field is present.

The ions are accelerated along the region where the electric field is not null in Fig. 7.3b and therefore the force $F_{T i}(z)$ increases up to the constant value in Fig. 7.4b as predicted by Eq. 7.1. Then, the ion acceleration region is limited up to $Z_{n} \simeq 20 \mathrm{~mm}$.

Finally, the thrust delivered can be estimated from the limit ion exhaust velocity of Fig. $7.3 \mathrm{a}$ as $F_{T i} \simeq 1.6 \mathrm{mN}$ in Fig. $7.4 \mathrm{~b}$ and the specific impulse $I_{s p}=v_{e x} / g_{o} \sim$ $1.43 \times 10^{4} \mathrm{~s}$. This value is one order of magnitude higher than those of Table 7.1.

\subsection{The Mesothermal Plasma Flow}

As we have seen, the multi-electrode structures of Figs. 7.1 and 7.2 might deliver ions at supersonic velocities of $v_{e x} \sim 140 \mathrm{~km} / \mathrm{s}$, much higher than the typical values of Table 7.1. The next step is to test in the laboratory under realistic conditions an optimized multi-electrode prototype.

In practical terms, the distribution of the ion axial velocity along the thrust axis of Figs. 7.1 and 7.2 is not monoenergetic. Since diverging ion flow in the plasma plume reduces the axial velocity component that provides the thrust, the efficiency of the multi-electrode systems requires of narrow velocity distributions.

This axial ion velocity distribution function (IVDF) can be directly measured in the laboratory and can provide a method of validation of numerical simulations discussed in the previous section. The four-grids retarded field energy analyzer (RFEA) technique can be used to characterize the low energy and supersonic ion populations in the plasma plume. The details of this diagnostic and our specific experimental setup are discussed in Ref. [15].

This RFEA operates under stationary conditions and its collector current $I_{c}\left(V_{i d}\right)$ is composed of ions with energy over the retarding voltage $V_{i d}$. The velocity distribution functions of ions at different points of the plasma plume are obtained from these $I_{c}\left(V_{i d}\right)$ current-voltage curves. In our case, the resolution for ions with speeds $u_{z}>0$ 
Fig. 7.5 The ion velocity distribution functions measured along the $Z$ axis of symmetry of a plasma thruster $[16,17]$.

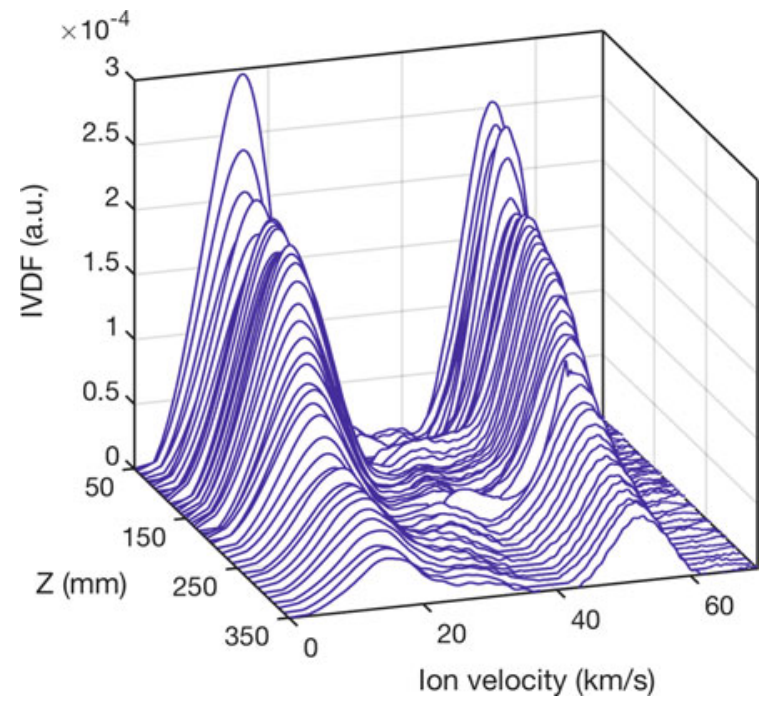

parallel to the RFEA axis of symmetry was about $\pm 8 \mathrm{eV}$ for singly charged Argon ions of $400 \mathrm{eV}$ energy. This is equivalent to radial speeds of $\pm 6 \mathrm{~km} / \mathrm{s}$ for ions with axial velocities of $44 \mathrm{~km} / \mathrm{s}$ [15].

Figure 7.5 show the characteristic IVDFs of a mesothermal plasma plume flow. The RFEA was located along the axis of symmetry of a plasma plume produced by a plasma thruster and the $I_{c}\left(V_{i d}\right)$ curves were recorded between 50 and $350 \mathrm{~mm}$ from its exit section, holding constant its parameters of operation [17].

The electron and ion distribution functions at a distant point were approximately Maxwellian with plasma densities $n_{e} \simeq 0.1-8.0 \times 10^{8} \mathrm{~cm}^{-3}$ with electron temperatures of $T_{e}=1-2 \mathrm{eV}$. The electron Debye lengths for this background plasma were of $\lambda_{D e} \simeq 0.2-0.3 \mathrm{~mm}$.

The waterfall representation of the two-peaked distributions in Fig. 7.5 shows an slow ion group with a maximum around $u_{s l} \simeq 15 \mathrm{~km} / \mathrm{s}$ superposed to a fast ion peak located at $u_{f s} \simeq 53 \mathrm{~km} / \mathrm{s}$. The shape of the IVDFs remains basically constant along 50-350 $\mathrm{mm}$ but the heights of maxima decrease with the axial coordinate $Z$ as the plasma stream expands, reflecting the decreasing axial plasma density. The low energy group is insensitive to the control parameters of the thruster whereas the maxima of the high energy group depends on the thruster throttle potential.

The temperatures of both ion groups where estimated from the full width at half maximum of the IVDFs in Fig. 7.5 and give similar values along the $Z$ axis of $T_{i s l} \simeq$ $30 \mathrm{eV}$ for the low-energy ion group and $T_{i f s} \simeq 120 \mathrm{eV}$ for the fast-ion population. Hence, the fast ion group drifts with a typical supersonic speed of $u_{D}=u_{f s}-u_{s l} \sim$ $38 \mathrm{~km} / \mathrm{s}$ much higher than the ion sound speed of $c_{i s} \sim 8.5 \mathrm{~km} / \mathrm{s}$ for this group. The plasma plume is therefore a mesothermal flow where ions drifts at supersonic speeds.

The origin of the slow ion group would be the finite size of the vacuum tank. The cross sections for elastic and resonant charge exchange collisions of ions with neutral 
atoms give mean free paths of $\lambda_{a i} \simeq 500 \mathrm{~cm}$ for our working pressures and we also have $\lambda_{a a} \simeq 430 \mathrm{~cm}$. As the size of our vacuum chamber is smaller, collisions in the plasma plume do not explain this low energy ion group.

The interaction of fast ions with the walls of the vacuum tank in the steady state is the more likely origin. A fraction recombines at the walls and the other group loose energy and is reflected back with reduced velocities pointing along random directions after colliding. This explains the lack of response of the low ion group in Fig. 7.5 to throttle voltage and its distribution as an uniform background in the steady state [15-17]. A similar response can be expected for the multi-electrode structures of Figs. 7.1 and 7.2 along the thrust axis $Z$.

\subsection{Conclusions and Outlook}

The combination of analytical numerical simulations using CST software is fast and flexible numerical scheme for the design and optimization of multi-electrode structures intended to accelerate ions to supersonic velocities. Analysis of the simulation data shows the adequacy and consistency of this approach. Similar methods can be adapted to study alternative ion acceleration configurations and/or plasma thrusters. Such as Hall-effect or gridded ion engines [10-14] using affordable computational resources.

The numerical simulations suggests the small the multi-electrode configuration of Figs. 7.1 and 7.2 might accelerate ions up to exhaust velocities in the order of hundred of kilometers per second as shows Fig. 7.3a. This represents one order of magnitude faster than usual plasma thrusters, such as Hall-effect of gridded ion engines [1,2]. Consequently, ion currents as low as $I_{i}=14 \mathrm{~mA}$ can give rise thrusts in the millinewton range leading to elevated specific impulses.

An advantage of the electrode configurations in Figs.7.1 and 7.2 is their small size and weight, but unfortunately they require high voltages to operate. Electrical insulation and power consumption can be an issue.

For optimum particle collimation the charge losses at the exposed surface of electrodes along the acceleration channel can be reduced to a minimum. The electrode polarization power supplies do not consume electrical energy when the drained current is null. This would reduce the weight and electric power budget of the system. Additional numerical simulations with improved multiple electrode designs are required to meet this objective.

Finally, the mesothermal plasma flow exhaust can be characterized using standard RFEA equipment. The numerical simulations can be assessed also using the axial ion velocity distribution functions to improve the collimation of ion flow and hence the delivered thrust.

Acknowledgements This research was funded by Peter the Great St. Petersburg Polytechnic University (SPbPU) in the framework of program 5-100-2020. Additionally, this paper was partially supported by the Ministry of Science, Innovation and Universities of Spain under Grant 
RT2018-094409-B-100. The invitation by SPbPU University to one of us (LC) in the summer 2019 is also gratefully acknowledged.

\section{References}

1. D.M. Goebel, I. Katz, Fundamentals of Electric Propulsion. Ion and Hall Thrusters (Wiley, Hoboken, 2008)

2. S. Mazouffre, Electric propulsion for satellites and spacecraft: established technologies and novel approaches. Plasma Sources Sci. Technol. 25(3), 033002-1,27 (2016)

3. C. Charles, Plasmas for spacecraft propulsion. J. Phys. D: Appl. Phys. 42(16), R1 163001-1,18 (2009)

4. I. Levchenco et al., Perspectives, frontiers, and new horizons for plasma-based space electric propulsion. Phys. Plasmas 27(2), 020601-1,30 (2020)

5. F.F. Chen, Introduction to Plasma Physics and Controlled Fusion, vol. 1 (Plenum Press, New York, 1984)

6. N. Koch et al., The HEMPT concept. A survey on theoretical considerations and experimental evidences, in Proceedings of the 32th International Electric Propulsion Conference, IEPC paper 2011-236, Weisbaden, Germany, 11-15 September 2011

7. E. Boch, The HEMPT NG, in Proceedings of the Electric Propulsion, Innovation \& Competitiveness Workshop, Madrid, Spain, 24-25 October 2017

8. Computer Simulation Technology (CST) Studio Suite. Electromagnetic Field Simulation Software. Dassault Systémes. https://www.3ds.com/products-services/simulia/products/cststudio-suite/

9. D. Dyubo, O.Yu. Tsybin, A computer model of the contact ionization ion accelerator for the electrically powered spacecraft propulsion. St. Petersburg State Polytechnical Univ. J. Phys. Math. 13(1), 78-91 (2020)

10. A. Hassan, A. Elsaftawy, S.G. Zakhary, Analytical studies of the plasma extraction electrodes and ion beam formation. Nuclear Instrum. Meth. A 586(2), 148-152 (2008)

11. O. Kalentev, K. Matyash, J. Duras, K.F. Lüskow, R. Schneider, N. Koch, M. Schirra, Electrostatic ion thrusters - towards predictive modeling. Contrib. Plasma Phys. 54(2) 235-248 (2014)

12. A.S. Lovtsov, D.A. Kravchenko, Kinetic simulation of plasma in ion thruster discharge chamber. Comparison with experimental data. Procedia Eng. 185, 326-331 (2017)

13. X. Peng, D. Keefert, W.M.P. Ruytent, Plasma particle simulation of electrostatic ion thrusters. J. Prop. Power 8(2), 361-366 (1992)

14. O.Y. Tsybin, S.B. Makarov, O.N. Ostapenko, Jet engine with electromagnetic field excitation of expendable solid-state material. Acta Astronaut. 129, 211-213 (2016)

15. J. Damba et al., Multiprobe characterization of plasma flows for space propulsion. J. Phys. Conf. Ser. 958, 012002-1,9 (2018)

16. L. Conde et al., Supersonic plasma beams with controlled speed generated by the alternative low power hybrid ion engine (ALPHIE) for space propulsion. Phys. Plasmas 24(4), 123514-1,8 (2017)

17. L. Conde et al., Physics and performance of the alternative low power hybrid ion engine (ALPHIE) for space propulsion, in Proceedings of the 32th International Electric Propulsion Conference, IEPC paper 2019-A-643, Vienna, Austria, 15-20 September 2019 